\title{
Control of Polydimethylsiloxane Surface Hydrophobicity by Plasma Polymerized Hexamethyldisilazane Deposition
}

\author{
Virginija Jankauskaitè ${ }^{1, *}$, Pranas Narmontas ${ }^{2}$ and Algirdas Lazauskas ${ }^{2}$ \\ 1 Department of Production Engineering, Kaunas University of Technology, Studentu St. 56, \\ LT-51424 Kaunas, Lithuania \\ 2 Institute of Materials Science, Kaunas University of Technology, Barsausko St. 59, LT-51423 Kaunas, \\ Lithuania; pranas.narmontas@ktu.lt (P.N.); algirdas.lazauskas@ktu.lt (A.L.) \\ * Correspondence: virginija.jankauskaite@ktu.lt; Tel.: +370-610-05-190
}

Received: 30 November 2018; Accepted: 10 January 2019; Published: 11 January 2019

\begin{abstract}
The properties of a polydimethylsiloxane (PDMS) surface were modified by a one-step deposition of plasma polymerized hexamethyldisilazane (pp-HMDS) by the arc discharge method. Scanning electron microscopy, atomic force microscopy, and Fourier-transform infrared spectroscopy analytical techniques were employed for morphological, structural, and chemical characterization of the pp-HMDS modified PDMS surface. The changes in PDMS substrate wetting properties were evaluated by means of contact angle measurements. The unmodified PDMS surface is hydrophobic with a contact angle of $122^{\circ}$, while, after pp-HMDS film deposition, a dual-scale roughness PDMS surface with contact angle values as high as $170^{\circ}$ was obtained. It was found that the value of the contact angle depends on the plasma processing time. Chemically, the pp-HMDS presents methyl moieties, rendering it hydrophobic and making it an attractive material for creating a superhydrophobic surface, and eliminating the need for complex chemical routes. The presented approach may open up new avenues in design and fabrication of superhydrophobic and flexible organosilicon materials with a self-cleaning function.
\end{abstract}

Keywords: polydimethylsiloxane; superhydrophobicity; hexamethyldisilazane; plasma polymerization

\section{Introduction}

Polydimethylsiloxanes (PMDS) are the most widely used silicon-based organic polymers, commonly referred to as silicones. Because of easy fabrication, non-toxicity, biocompatibility and biodurability they have found potential applications in various fields. The surface of PDMS is naturally hydrophobic, but a number of efforts have been made to modify PDMS and further enhance its hydrophobicity [1,2]. PDMS hydrophobicity plays an important role in diverse applications e.g., self-cleaning surfaces [3], microfluidics [4], microelectromechanical systems [5], and biomedical applications [6].

Superhydrophobic PDMS surfaces can be fabricated by pulsed laser irradiation resulting in surface modification with a static contact angle (CA) value of $170^{\circ}$ [7]. However, the whole irradiation procedure is highly time-consuming, thus limiting the scalability of this method. A more reliable and effective practice includes the deposition/formation of a thin film on the surface of the material to obtain the desired functionality. Plasma treatment is attractive as the processing time is short, the process involves low temperature, and procedures are relatively simple. Importantly, a single-step technique is desired for obtaining superhydrophobic and self-cleaning surface functionalities.

In this contribution, we fabricated a superhydrophobic PDMS surface via plasma polymerized hexamethyldisilazane (pp-HMDS) thin film deposition by arc discharge. To the best of our knowledge, 
the technique adopted here has been not reported for the fabrication of superhydrophobic PDMS surface using hexamethyldisilazane monomer as a precursor. HMDS is well known as being widely used for hydrophobic coatings on various hydroxyl-bearing surfaces [8,9]. HMDS chemical activity derives from the presence of a highly reactive nitrogen atom within the compound. The presented one step deposition of in situ polymerized hexamethyldisilazane is simple and scalable, and thus can provide a new strategy for the large scale fabrication of superhydrophobic surfaces with a self-cleaning function on flexible substrates.

\section{Materials and Methods}

The addition-curing silicone rubber Elastosil RT $601 \mathrm{~A} / \mathrm{B}$ with a viscosity of $3500 \mathrm{mPa} \cdot \mathrm{s}$ at $23{ }^{\circ} \mathrm{C}$ (Wacker Chemie AG, Múnich, Germany) was used as received for flexible films fabrication. HMDS of analytical grade ( $\geq 99 \%$, Sigma-Aldrich, Saint Louis, MO, USA) was used as received.

The experimental setup of arc plasma reactor and technological conditions have been reported previously [10]. Briefly, a rod-shaped graphite anode and cathode were placed at the center of the discharge chamber. A quartz cuvette containing HMDS solution was positioned $20 \mathrm{~mm}$ from the anode, and PDMS substrate was placed at a distance of $15 \mathrm{~mm}$ from the electrodes. The chamber was connected to a vacuum line backed by a rotary pump. Arc-discharge was generated between anode and cathode using a DC transferred arc process using $\sim 4.3 \mathrm{~mA}$ current and $\sim 25 \mathrm{kV}$ voltage. The deposition time was varied up to $60 \mathrm{~s}$.

A FEI Quanta 200 scanning electron microscope (SEM, Thermo Fisher Scientific, Waltham, MA, USA) was used to collect micrographs of the investigated surface. The samples were imaged at an accelerating voltage of $30 \mathrm{kV}$. Atomic force microscopy (AFM) experiments were carried out with NT-206 (Microtestmachines, New Taipei City, Taiwan) in air at room temperature $\left(22 \pm 1^{\circ} \mathrm{C}\right)$ using a V-shaped silicon cantilever operating in contact mode. The surface morphology of the resulting films was evaluated based on the AFM surface topography images and roughness parameters. Vertex 70 Fourier transform infrared (FTIR) spectrometer (Bruker Optics Inc., Billerica, MA, USA) equipped with a 30Spec (Pike Technologies, Madison, IA, USA) specular reflectance accessory having a fixed $30^{\circ}$ angle of incidence was used for the chemical characterization of the modified PMDS surface.

CA measurements were performed at room temperature using the sessile drop method. A droplet of deionized water $(5 \mu \mathrm{L})$ was deposited onto the investigated surface. Optical images of the droplet were recorded with a PC-connected digital camera after $10 \mathrm{~s}$ of dropping and CA measurements were carried out using an active contour method based on B-spline snakes (active contours) [11]. The contact angle hysteresis was measured as the difference between the advancing and receding contact angle of a sliding droplet. The test was performed by setting a droplet on a sample, which was placed on a horizontal plate. The plate was tilted slowly until the water drop began to slide along the surface; at this point the camera shutter was activated. The advancing and receding contact angles where then measured.

\section{Results}

The morphology of unmodified and plasma polymerized HMDS (pp-HMDS) modified flexible PDMS substrate and water droplets on the PDMS surface before and after pp-HMDS film deposition at different times are compared in Figure 1. Cured PDMS is produced spontaneously forming wavy structures on the surface with micro-scale amplitude and periodicity of $128 \mathrm{~nm}$. SEM images of the resulting pp-HDMS thin film surface for deposition times of 30 and $60 \mathrm{~s}$ are presented in Figure $1 b, c$, respectively. The deposition resulted in a highly branched and crosslinked pp-HMDS structures composed of quasi-spherical nanoparticles with size in the range of $15-60 \mathrm{~nm}$. Growth in three-dimensional assembles and formation of large nanoparticles aggregates were observed as the deposition time increased.

These morphological alterations change the wetting properties of the PDMS surface (Figure 1). The unmodified PDMS surface exhibits hydrophobic behavior with a static CA value not higher than $122^{\circ}$. 
A considerable improvement in non-wetting characteristics of pp-HMDS film functionalized surfaces was observed. After $30 \mathrm{~s}$ of deposition, the nanostructured pp-HMDS film exhibited superhydrophobic properties with static $C A$ values of $169^{\circ}-170^{\circ}$. In this case the low value of $C A$ hysteresis $\left(2^{\circ}\right)$, defined as the difference between the CA at the front of the droplet (advancing CA) and at the back of the droplet (receding CA), was obtained. The increase of deposition times up to $60 \mathrm{~s}$ results in lower CA values, i.e., $\mathrm{CA}=159^{\circ}-161^{\circ}$.
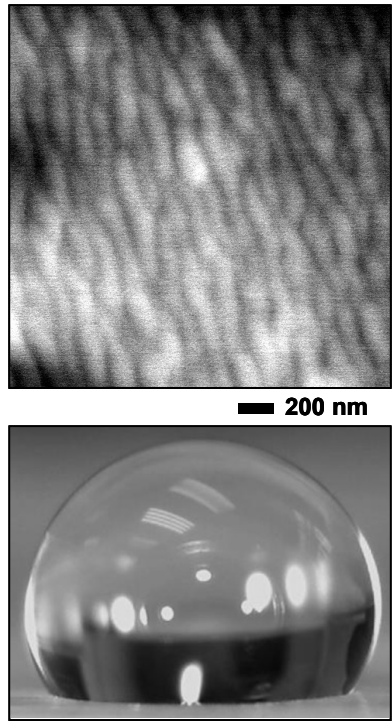

$\mathrm{CA}=118-122^{\circ}$

(a)
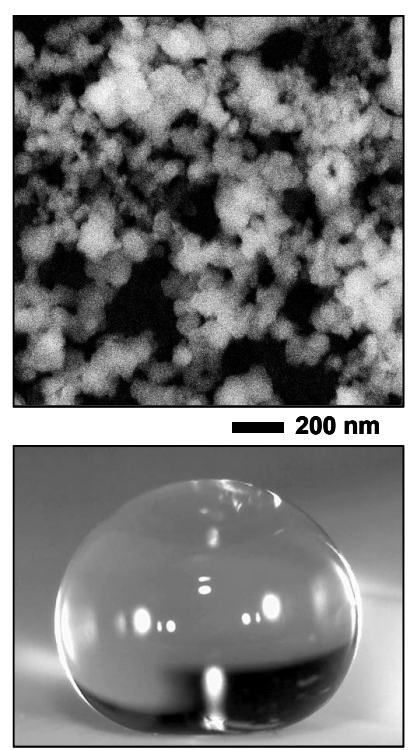

$C A=169-170^{\circ}$

(b)
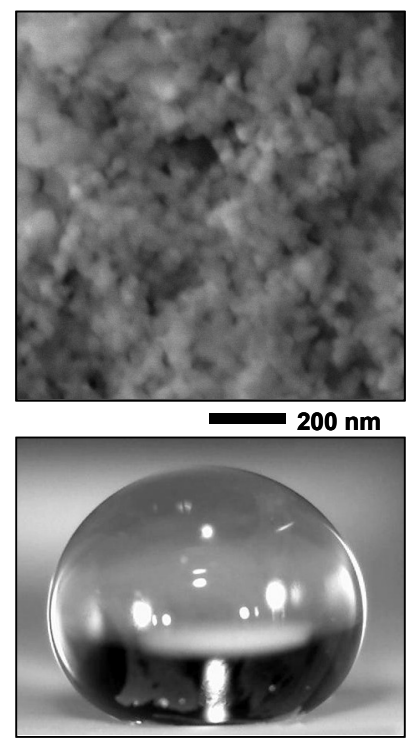

$C A=159-161^{\circ}$

(c)

Figure 1. SEM image of unmodified (a) and pp-HMDS film modified PDMS surfaces $(\mathbf{b}, \mathbf{c})$ and water droplets on PDMS surface before and after pp-HMDS film deposition at different times: (a) 0; (b) 30 s; (c) $60 \mathrm{~s}$.

The presence of nanostructures in the form of quasi-spherical nanoparticles and interconnection caused by the formation of large aggregates at longer pp-HMDS deposition time (60 s) was confirmed using characteristic AFM topographical and surface profile images shown in Figure 2. In this case dual-scale roughness of the surface was maintained, the pp-HMDS film surface was found to be rough with the root-mean square roughness having a value of $96.11 \mathrm{~nm}$. However, the spiky surface morphology changes into a bumpy one and the negative surface skewness parameter value $(-0.2)$ indicates predominance of valleys.

As can be seen from Figure 3a, in the FTIR absorbance spectrum of unmodified PDMS the bands at 2965 and $2906 \mathrm{~cm}^{-1}$ are assigned to asymmetric and symmetric stretching of $\mathrm{CH}_{3}$ groups, respectively [12]. The asymmetric and symmetric bending vibrations of $\mathrm{CH}_{3}$ groups are also observed at 1410 and $1258 \mathrm{~cm}^{-1}$, respectively. The bands at 1072 and $1007 \mathrm{~cm}^{-1}$ are characteristic of Si-O-Si asymmetric and symmetric stretching vibrations, respectively. Asymmetric rocking at $864 \mathrm{~cm}^{-1}$ and stretching at $785 \mathrm{~cm}^{-1}$ vibrations can be attributed to the $\mathrm{Si}-\mathrm{CH}_{3}$ group [12]. 


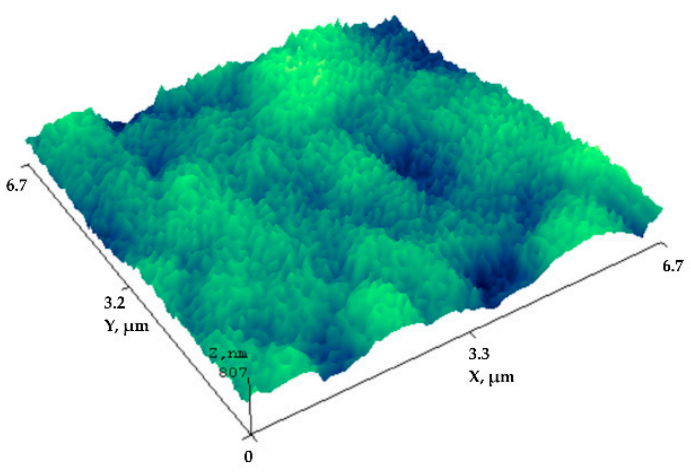

(a)

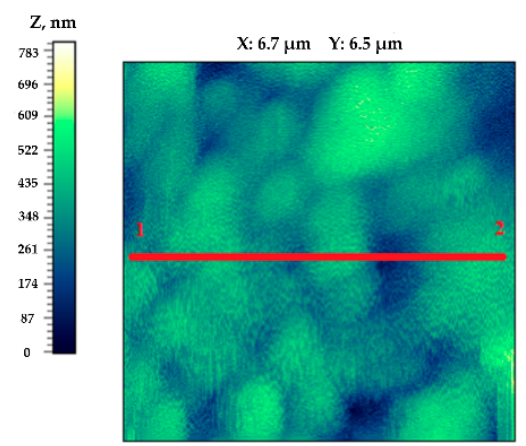

(b)

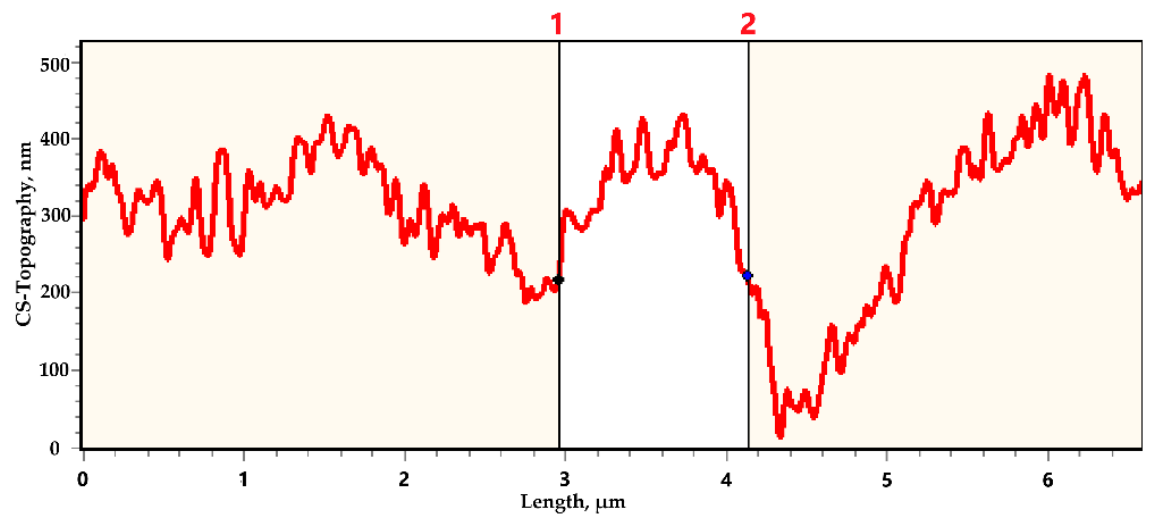

(c)

Figure 2. AFM image of 3D (a) and 2D (b) topography with normalized Z (nm scale), and profilogram of pp-HMDS film at deposition time of $60 \mathrm{~s}$ (c).

The deposition of polymerized pp-HMDS film leads to the obvious PDMS surface functional group changes (Figure $3 \mathrm{~b}$ ). The broad band between 3400 and $3700 \mathrm{~cm}^{-1}$ is related to O-H stretching in Si-OH bonds of hydrophilic silanol groups [13]. The absorbance at $3350 \mathrm{~cm}^{-1}$ is characteristic for stretching of the N-H bond, while the doublet at $2350 \mathrm{~cm}^{-1}$ is attributed to $\mathrm{CO}_{2}$ species [10]. As in the case of unmodified PDMS, the presence of methyl moieties in the modified surface is confirmed by an absorption band at $1410 \mathrm{~cm}^{-1}$, related to $\mathrm{CH}_{3}$ asymmetric bending in $\mathrm{Si}-\mathrm{CH}_{3}$ bonds, and 2965 and $2906 \mathrm{~cm}^{-1}$ bands, which are characteristic for asymmetric stretching and symmetric stretching of the $\mathrm{CH}_{3}$ group, respectively $[14,15]$. A low intensity band at $1454 \mathrm{~cm}^{-1}$ is assigned to the asymmetric bending vibrations of the $\mathrm{CH}_{2}$ group in the $\mathrm{Si}-\mathrm{CH}_{2}-\mathrm{CH}_{2}-\mathrm{Si}$ link that play a substantial role in the cross-linking process during HMDS polymerization [15]. The band located at $2250 \mathrm{~cm}^{-1}$ corresponds to $\mathrm{Si}-\mathrm{H}$ stretching vibration [16], while the band at $1629 \mathrm{~cm}^{-1}$ can be assigned to stretching of $\mathrm{C}=\mathrm{O}$ [17]. Some oxygen related functional groups could arise from free radical (possibly trapped in the film structure) reaction with the atmosphere, when the samples are removed from the reactor [18]. 


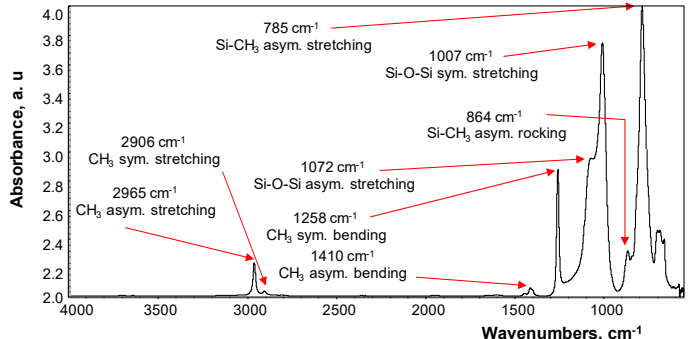

(a)

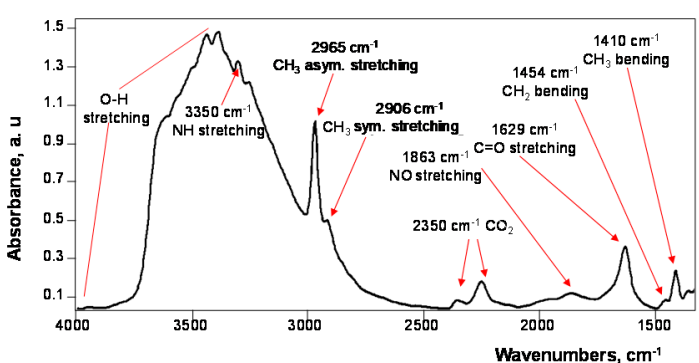

(b)

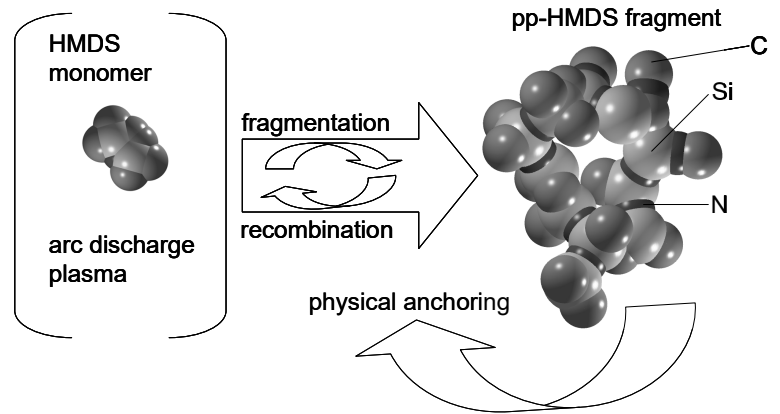

(c)

Figure 3. FTIR absorbance spectra of flexible PDMS substrate (a) and pp-HMDS (b) with functional groups assigned and schematic diagram of probable deposition mechanism (c); HMDS monomer and pp-HMDS network fragment are shown in van der Waal's-based representation.

\section{Discussions}

Generally, the hydrophobic properties of films are determined by the kind and amount of grafted hydrophobic groups and surface roughness parameters. One step coating by in situ HMDS deposition and polymerization is an easy and rapid method to impart non-wetting properties to the PDMS surface. The plasma polymerization process of HMDS monomers resulted in highly branched and crosslinked structures composed of quasi-spherical nanoparticles. After pp-HMDS film deposition, the PDMS surface shows superhydrophobic characteristics with a CA value close to $170^{\circ}$. High hydrophobicity of pp-HMDS originates from the high amount of $\mathrm{CH}_{3}$ species and specific film surface morphology. The pp-HMDS film functionalized surfaces exhibited Cassie-Baxter state with a "lotus effect" observable and a low CA hysteresis of $2^{\circ}$, suggesting that a water droplet is not able to wet the spaces between surface morphological features allowing air pockets to remain at the interface. The increase of pp-HMDS film deposition time influences the decrease of CA value. It can be attributed to a higher solid fraction of surface morphological features in contact with the water droplet, which decreases the concentration of air pockets trapped at the interface of pp-HMDS with the droplet.

Based on the SEM, FTIR data, and surface wetting studies, it is suggested that the HDMS monomer molecules passing to the arc plasma region during the operational process are fragmented with partial retention and formation of new chemical bonds. The corresponding repetition of fragmentation and recombination reactions of HMDS monomers in arc plasma leads to the deposition of a randomly crosslinked network structure of pp-HMDS (Figure 3c) and forms a heterogeneous surface with a high fraction of methyl moieties retained, thus providing superhydrophobic characteristics with a self-cleaning function.

Thus, FTIR investigations reveal multiple non-covalent interaction achieved by in situ HMDS polymerization with physical anchoring on the polymer surface [19]. Such an interaction can be recognized as the driving force for constructing and fabrication of superhydrophobic and flexible organosilicon materials with a self-cleaning function. 


\section{Conclusions}

Herein, we successfully enhanced the non-wetting properties of a flexible polydimethylsiloxane substrate surface via plasma polymerized hexamethyldisilazane thin film deposition by the arc discharge method. Such a film is composed of quasi-spherical nanoparticles stacked together, which form a branched network. The deposited nanostructured plasma polymerized hexamethyldisilazane film exhibits superhydrophobic properties with static contact angle values as high as $170^{\circ}$ and a low contact angle hysteresis of $2^{\circ}$. The PDMS surface undergoes self-cleaning and non-wetting behavior due to the multiple non-covalent interactions attended by the incorporation in the surface layer of methyl groups and a nano-rough surface formation. This is a facile and effective method that can provide a new strategy for the large scale fabrication of superhydrophobic surfaces with a self-cleaning function on flexible substrates.

Author Contributions: Conceptualization, A.L. and V.J.; Methodology, A.L. and P.N.; Validation, V.J. and P.N.; Formal Analysis, V.J.; Investigation, A.L. and P.N.; Resources, A.L. and V.J.; Writing-Original Draft Preparation, V.J. and A.L.; Writing-Review \& Editing, V.J. and A.L.; Visualization, A.L. and V.J.; Supervision, V.J.; Project Administration, A.L.; Funding Acquisition, V.J.

Funding: This research was funded by the European Social Fund under "Development of Competences of Scientists, other Researchers and Students through Practical Research Activities" measure (No. 09.3.3-LMT-K-712-01-0074).

Conflicts of Interest: The authors declare no conflict of interest.

\section{References}

1. Yang, J.; Pi, P.; Wen, X.; Zheng, D.; Xu, M.; Cheng, J.; Yang, Z. A novel method to fabricate superhydrophobic surfaces based on well-defined mulberry-like particles and self-assembly of polydimethylsiloxane. Appl. Surf. Sci. 2009, 255, 3507-3512. [CrossRef]

2. Slaughter, G.; Stevens, B. A cost-effective two-step method for enhancing the hydrophilicity of PDMS surfaces. BioChip J. 2014, 14, 28-34. [CrossRef]

3. Tavares, M.T.S.; Santos, A.S.F.; Santos, I.M.G.; Silva, M.R.S.; Bomio, M.R.D.; Longo, E.; Paskocimas, C.A.; Motta, F.V. $\mathrm{TiO}_{2} /$ PDMS nanocomposites for use on self-cleaning surfaces. Surf. Coat. Technol. 2014, 239, 16-19. [CrossRef]

4. Tropmann, A.; Tanguy, L.; Koltay, P.; Zengerle, R.; Riegger, L. Completely superhydrophobic PDMS surfaces for microfluidics. Langmuir 2012, 28, 8292-8295. [CrossRef] [PubMed]

5. Basu, B.J.; Bharathidasan, T.; Anandan, C. Superhydrophobic oleophobic PDMS-silica nanocomposite coating. Surf. Innov. 2013, 1, 40-51. [CrossRef]

6. Martin, S.; Bhushan, B. Transparent, wear-resistant, superhydrophobic and superoleophobic poly(dimethylsiloxane) (PDMS) surfaces. J. Colloid Interface Sci. 2017, 488, 118-126. [CrossRef] [PubMed]

7. Khorasani, M.T.; Mirzadeh, H. In vitro blood compatibility of modified PDMS surfaces as superhydrophobic and superhydrophilic materials. J. Appl. Polym. Sci. 2004, 91, 2042-2047. [CrossRef]

8. Terpilowski, K.; Goncharuk, O. Hydrophobic properties of hexamethyldisilazane modified nanostructured silica films on glass: Effect of plasma pre-treatment of glass and polycondensation features. Mater. Res. Express 2018, 5, 016409. [CrossRef]

9. Protsak, I.; Pakhlov, E.; Tertykh, V.; Le, Z.-C.; Dong, W. A new route for preparation of hydrophobic silica nanoparticles using a mixture of poly(dimethylsiloxane) and diethyl carbonate. Polymers 2018, 10, 116. [CrossRef]

10. Lazauskas, A.; Baltrusaitis, J.; Grigaliūnas, V.; Jucius, D.; Guobienè, A.; Prosyčevas, I.; Narmontas, P. Characterization of plasma polymerized hexamethyldisiloxane films prepared by arc discharge. Plasma Chem. Plasma Process. 2014, 34, 271-285. [CrossRef]

11. Stalder, A.; Kulik, G.; Sage, D.; Barbieri, L.; Hoffmann, P. A snake-based approach to accurate determination of both contact points and contact angles. Colloid Surf. A 2006, 286, 92-103. [CrossRef]

12. Cai, D.; Neyer, A.; Kuckuk, R.; Heise, H.M. Raman, mid-infrared, near-infrared and ultraviolet-visible spectroscopy of PDMS silicone rubber for characterization of polymer optical waveguide materials. J. Mol. Struct. 2010, 976, 274-281. [CrossRef] 
13. Tielens, F.; Gervais, C.; Lambert, J.F.; Mauri, F.; Costa, D. Ab initio study of the hydroxylated surface of amorphous silica: A representative model. Chem. Mater. 2008, 20, 3336-3344. [CrossRef]

14. Grimoldi, E.; Zanini, S.; Siliprandi, R.; Riccardi, C. AFM and contact angle investigation of growth and structure of pp-HMDSO thin films. Eur. Phys. J. D 2009, 54, 165-172. [CrossRef]

15. Benitez, F.; Martınez, E.; Esteve, J. Improvement of hardness in plasma polymerized hexamethyldisiloxane coatings by silica-like surface modification. Thin Solid Films 2000, 377, 109-114. [CrossRef]

16. Anderson, R.C.; Muller, R.S.; Tobias, C.W. Chemical surface modification of porous silicon. J. Electrochem. Soc. 1993, 140, 1393-1396. [CrossRef]

17. Yamada, T.; Lukac, P.J.; Yu, T.; Weiss, R.G. Reversible, room-temperature, chiral ionic liquids. Amidinium carbamates derived from amidines and amino-acid esters with carbon dioxide. Chem. Mater. 2007, 19, 4761-4768. [CrossRef]

18. Honda, R.Y.; Mota, R.P.; Batocki, R.; Santos, D.; Nicoleti, T.; Kostov, K.; Kayama, M.; Algatti, M.; Cruz, N.; Ruggiero, L. Plasma-polymerized hexamethyldisilazane treated by nitrogen plasma immersion ion implantation technique. J. Phys. Conf. Ser. 2009, 167, 012055. [CrossRef]

19. Wei, Q.; Haag, R. Universal polymer coatings and their representative biomedical applications. Mater. Horiz. 2015, 2, 567-577. [CrossRef]

(C) 2019 by the authors. Licensee MDPI, Basel, Switzerland. This article is an open access article distributed under the terms and conditions of the Creative Commons Attribution (CC BY) license (http://creativecommons.org/licenses/by/4.0/). 